\title{
Aneurisma de artéria poplítea como manifestação da doença de Behçet descompensada
}

\author{
Popliteal artery aneurysm as a manifestation of decompensated Behçet's disease
}

\author{
Gabriel Salum D 'Alessandro, Rafael Forti M achietto, Simone M artins da Silva, Walter Campos Jr., \\ Cláudio Jamil Akel, Renato Murilo Etchebehere, Roberta M urasaki Cardoso, N ilo M itsuru Izukawa*
}

\begin{abstract}
Resumo
A doença de Behçet consiste em uma vasculite sistêmica que acomete veias, artérias e capilares, mas predominantemente pequenos vasos. Raramente há o envolvimento de veias e artérias de médio e grande calibre, sendo as artérias menos acometidas que as veias. $\mathrm{No}$ presente trabalho, os autores apresentam um relato de caso de aneurisma da artéria poplítea em um pacientecom a doença de Behçet descompensada. D iscutem-se os aspectos clínicos e radiológicos e o manejo cirúrgico dos pacientes com essa doença inflamatória sistêmica que apresentam peculiaridades importantes.
\end{abstract}

Palavras-chave: Síndrome de Behçet, aneurisma, artéria poplítea.

\section{Relato de caso}

Paciente branco, 51 anos, sexo masculino, queixava-se de dor e presença de tumoração pul sátil em região posterior de coxa esquerda há 1 dia da data de internação. Referia, também, presença de úlceras em cavidade oral e febre vespertina há 2 meses.

0 paciente, com história de doença de Behçet diagnosticada em 1999, fazia uso irregular de ciclosporina e colchicina. 0 diagnóstico tinha sido feito com base no quadro de úlceras bipolares orais e genitais, eritema nodoso, uveíte anterior e aneurisma em artéria poplítea direita. Este, na época, foi corrigido através de aneurismectomia e enxerto fêmoro-poplíteo, com pró-

\footnotetext{
* Serviço de Angiologia e Cirurgia Vascular, Hospital Professor Edmundo Vasconcelos, São Paulo, SP.

Artigo submetido em 12.04.06, aceito em 18.07.06.
}

J Vasc Bras 2006;5(3):215-9.

Copyright $\odot 2006$ by Sociedade Brasileira de Angiologia e de Cirurgia Vascular.

\begin{abstract}
B ehçet's disease consists of a systemic vasculitis that affects veins, arteries and capillaries, but predominantly small vessels. Veins and medium- and large-caliber arteries are rarely involved; however, arteries are less involved than veins. In this paper the authors present a case of popliteal artery aneurysm in a patient with decompensated Behçet's disease. We discuss clinical, radiological and surgical aspects of patients with this inflammatory systemic disease who present important peculiarities.
\end{abstract}

Key words: Behçet's syndrome, aneurysm, popliteal artery.

tese de politetrafluoretileno (PTFE) de $6 \mathrm{~mm}$, tendo evoluído com obstrução do enxerto no oitavo mês de seguimento, sem desenvolvimento de quadro isquêmico grave, e sendo adotado tratamento clínico.

Ao exame clínico, apresentava-se em bom estado geral, corado, hidratado e febril $(38,2 \stackrel{\circ}{\circ})$ ), com pressão arterial 130/60 mmH g em ambos os braços, freqüência respiratória de 18 incursões por minuto e freqüência cardíaca de 84 batimentos por minuto. 0 s exames cardiopulmonar e abdominal encontravam-se dentro dos padrões da normalidade. 0 exame do membro inferior esquerdo evidenciou massa pulsátil em cavo poplíteo e presença de pulsos distais, sendo ausentes os pulsos poplíteo, tibial anterior e posterior no membro contra-lateral. $\mathrm{Na}$ inspeção da cavidade oral, foram visualizadas ulcerações na mucosa.

Foi realizado eco-D oppler arterial demembro inferior esquerdo, que evidenciou dilatação aneurismática da artéria poplítea em sua porção proximal, medindo 
aproximadamente $5 \times 4 \times 6 \mathrm{~cm}$, com presença de trombo mural (Figura 1). 0 hemograma evidenciou leucocitose de 19.500/ $\mathrm{mm}^{1}$ (3\% bastonetes; $83 \%$ segmentados; $1 \%$ eosinófilos; $0 \%$ basófilos; $6 \%$ linfócitos; $7 \%$ monócitos) eplaquetasna marca de $333.000 / \mathrm{mm}^{1}$. A dosagem deproteínaC reativafoi de $7,1 \mathrm{mg} / \mathrm{dL}$ (valor de referência ou VR: $<6 \mathrm{mg} / \mathrm{dL}$ ), Alfal glicoproteína ácida $141 \mathrm{mg} / \mathrm{dL}$ (VR: 50-120 mg/dL), fibrinogênio $749 \mathrm{mg} / \mathrm{dL}$ (VR: 200-400 mg/dL) e velocidade de hemossedimentação $53 \mathrm{~mm}$ (VR: <10 minutos).

Inicialmente, foi realizada pulsoterapia com solumedrol $1 \mathrm{~g}$ ao dia por 3 diasecicl ofosfamida $1 \mathrm{~g}$ em dose única, com o objetivo de diminuir a atividade inflamatória da doença antes da realização da cirurgia para correção do aneurisma.

0 estudo arteriográfico evidenciou dilatação sacular póstero-lateral na artéria poplítea supragenicular e artérias distais pérvias (Figura 2).

Apósa pulsoterapia, o pacienteevoluiu com melhora da febre e do restante do quadro clínico, optando-se pela pronta correção cirúrgica do aneurisma. Realizouseaneurismectomia ereconstrução arterial com enxerto fêmoro-poplíteo supragenicular, com prótese dePT FE de $6 \mathrm{~mm}$ (Figura 3). N o intra-operatório, constatou-se trombose de veia poplítea justa-aneurismática, sendo realizado tratamento com heparina de baixo peso molecular, em dose plena, no pós-operatório imediato.

$\mathrm{N}$ o pós-operatório imediato, antes da al ta hospitalar, com o objetivo de diagnosticar outras lesões vascu- lares concomitantes, foram realizados duplex scan de membros superiores, carótidas e vertebrais e angiorressonância magnética de aorta torácica, abdominal e seus principais ramos, que não demonstraram qualquer anormalidade. 0 eco-D oppler-cardiograma colorido evidenciou insuficiência aórtica discreta, dilatação discreta da raiz da aorta e aorta descendente e fração de ejeção de $75 \%$.

0 paciente evoluiu afebril e sem intercorrências, recebendo alta clinicamente estável e com os pulsos distaispresentes. Asmedicações deal taforam prednisona, pulsoterapia de ciclofosfamida uma vez ao mês e antagonista de vitamina $\mathrm{K}$.

Após 5 meses de seguimento, o paciente continua com o mesmo esquema terapêutico, mantendo perfusão do membro inferior esquerdo, sem claudicação e pulsos distais presentes. D uplex scan arterial do membro inferior esquerdo evidenciou enxerto fêmoro-poplíteo com fluxo trifásico e velocidade normal.

\section{D iscussão}

A doença de Behçet foi primeiramente descrita por H uluse Behçet em 1937, caracterizada pela tríade clássica deúlceras aftosas orais recidivantes, úlceras genitais euveíteanterior ou iridociclite 2 . É uma doença crônica e multissistêmica, cuja etiologia ainda não é bem definida, mas especula-se que a auto-imunidade característica da doença seja desencadeada por infecções bacterianas, virais e por um componente ambiental ${ }^{1,3}$. Além
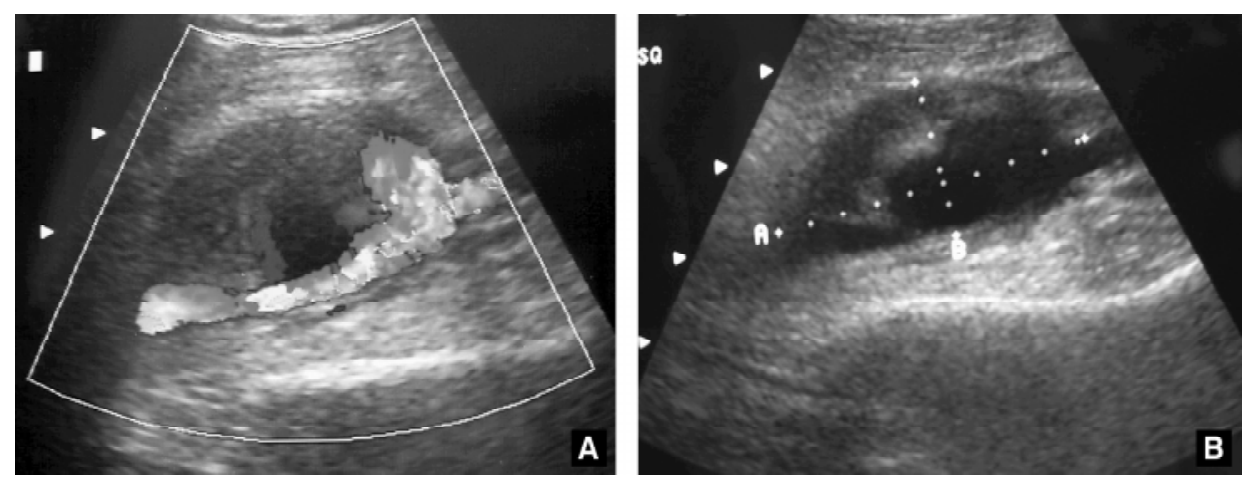

Figura 1 - A) D uplex scan arterial de membro inferior esquerdo evidenciando dilatação aneurismática da artéria poplítea em sua porção proximal; B) aneurisma medindo aproximadamente $5 \times 4 \times 6 \mathrm{~cm}$ com trombo mural 

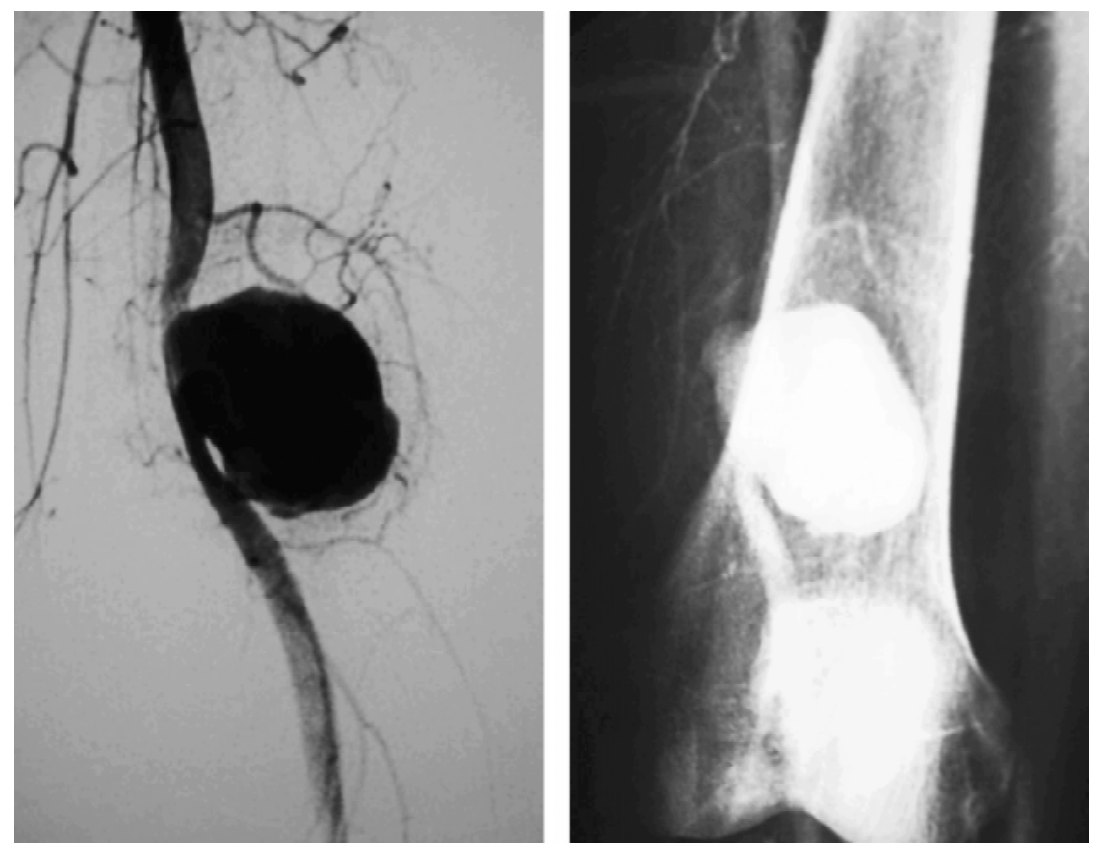

Figura 2 - Arteriografia evidenciando dilatação sacular póstero-lateral na arté ria poplítea supragenicular e artérias distais pérvias

disso, ésugerido um componentegenético, pelo fato de muitos pacientes apresentarem positividade para 0 $H \mathrm{LA}-\mathrm{B} 51^{4}$. Ela se desenvolve por volta da terceira à quarta década de vida e predomina em países do 0 riente $M$ édio, M editerrâneo e Ásia 0 riental ${ }^{5,6}$. Seu mecanismo fisiopatológico se caracteriza por uma vasculite sistêmica, que afeta veias, artérias e capilares ${ }^{1}$.
0 tratamento cirúrgico dessas complicações arteriais é caracterizado por grande risco de oclusão do enxerto e formação de pseudo-aneurisma. I sso ocorre devido à característica inflamatória local e sistêmica da doença?.

A doença de Behçet éconhecida como uma síndrome inflamatória crônica, multissistêmica, secundária à
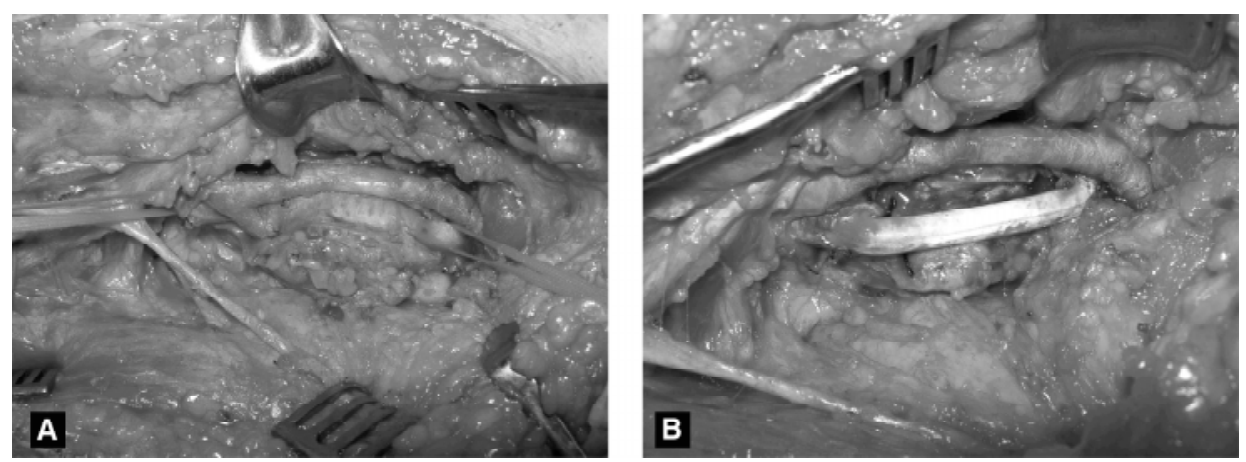

Figura 3 - A) Aneurisma da artéria poplítea adjacente à veia poplítea; B) correção do aneurisma com enxerto de politetrafluoretileno de $6 \mathrm{~mm}$ 
vasculite de pequenos vasos. A ausência de testes diagnósticos específicos torna difícil o diagnóstico da doença de Behçet, mesmo com os critérios clínicos diagnósticos propostos pelo grupo internacional de estudo sobre a doença de Behçet ${ }^{6}$ (T abela 1).

0 comprometimento predominante é nos peque nos vasos, podendo os vasos de médio e grande calibre serem afetados, respectivamente, em 9 e $27 \%{ }^{4}$. 0 comprometimento no sistema venoso é mais freqüente do que no arterial. As artérias são acometidas em 1,5 a $18 \%$ dos pacientes com doença de Behçet e geralmente como aneurismas em rápida expansão, com alto risco para rotura, o que torna a correção cirúrgica mandatória $^{5,8,9-14}$.

As lesões arteriais ocorrem com maior predomínio no sexo masculino, sendo o tabagismo um fator derisco importante ${ }^{15,16}$. N a maioria das vezes, ocorrem isoladamente, mas podendo comprometer múltiplos seguimentos arteriais e, freqüentemente, ser acompanhadas de trombose venosa ${ }^{17}$.

A patogenicidade da lesão aneurismática arterial é explicada por uma reação inflamatória envolvendo a camada média e adventícia do vaso. $\mathrm{H}$ istologicamente, os vasa vasorum encontram-se circundados por um intenso processo inflamatório leucocitário ${ }^{8,18}$. O s locais mais afetados são aorta e artéria pulmonar, seguidas pela artéria femoral, mas qualquer vaso de extremidades ou visceral pode estar envolvido, incluindo as artéria coronárias ${ }^{9,19,20}$. Por esse motivo, os pacientes com doença de Behçet devem ser rotineiramente investigados, à procura de aneurismas silenciosos, e sempre quando há ativação da doença. No caso apresentado, não havia outras lesões arteriais concomitantes, o quefoi comprovado por exames de imagem. C ontudo, havia trombose da veia poplítea diagnosticada no intra-operatório.

Pacientes com doença de Behçet associada ao acometimento arterial apresentam pior prognóstico quando comparados com pacientes sem comprometimento dasmesmas. A sobrevidarelatada naliteraturaéde $83 \%$ em 5 anos e $66 \%$ em 15 anos para aqueles com acometimento arterial $14,20,21$.

0 aneurisma arterial é mais comum do que a oclusão arterial e constitui importante causa de morte na doença de Behçet, secundária à ruptura do mesmo. Por esse motivo, o tratamento cirúrgico é obrigatório e deve ser feito o mais precocemente possível.

0 tratamento cirúrgico é freqüentemente complicado por recorrência do aneurisma, oclusão do enxerto e pseudo-aneurisma de anastomose ${ }^{22}$. Esses riscos aumentam muito quando a cirurgia é feita na fase ativa da doença. Por essemotivo, se possível, ela deveser postergada, devendo o tratamento antiinflamatório ser prontamente iniciado 7 . No presente caso, foi possível a realização de pulsoterapia com solumedrol eciclofosfamida antes do procedimento cirúrgico, com remissão satisfatória da atividade inflamatória.

$\mathrm{N}$ as revascularizações em pacientes portadores da doença de B ehçet, dá-se preferência aos enxertos sintéticos, devido ao processo inflamatório perivascular eà vasculite, que podem envolver as veias safenas ${ }^{7}$.

A terapiapós-operatória com corticosteróides, imunossupressores anticoagulantes e antiagregantes plaquetários tem sido sugerida como uma maneira eficaz para a prevenção da recorrência de aneurismas, oclusão do enxerto e ocorrência de pseudo-aneurismas anasto-

T abela 1 - Critérios diagnósticos da doença de Behçet

Critério diagnóstico D efinição

Úlceras orais recorrentes

Associado a dois ou mais casos de: Ú Iceras genitais recorrentes Lesões oculares Lesões de pele T este de patergia
U Icerações aftosas que recorrem $\geq$ três vezes em um período de 12 meses

Ú Icera aftosa ou escaras

U veíte anterior ou vasculite retiniana

Eritema nodoso, pseudofoliculite, lesões papulopustulosas

Leitura em 24-48 horas 
móticos $9,17,20,22-24$. N esteestudo, o pacientemantevese anticoagulado no pós-operatório recente com heparina de baixo peso molecular e encontra-se em uso de antagonistadavitaminaK paramanter arelação normalizada internacional (RNI) entre 2 e 4 . Além disso, encontra-se em uso de prednisona e é submetido à pulsoterapia mensal com ciclofosfamida.

\section{Referências}

1. Lakhanpal S, T ani K, LieJT, K atoh K, I shigatsubo Y, O hokubo $T$. Pathologic features of Behçet's syndrome: a review of Japanese autopsy registry data. H um Pathol. 1985;16:790-5.

2. Behçet $H$. Üeber rezidivierende Aphtose durch ein vírus verursachte Geschwüre am $\mathrm{M}$ und, am Auge und an den Genitalien. Dermatol Wochenschr. 1937;105:1152-7.

3. $H$ asan $A$, Fortune $F, W$ ilson $A$, et al. R ole of gamma delta $T$ cellsin pathogenesis and diagnosis of Behçet's disease. L ancet. 1996;347:789-94.

4. Suzuki Kurokawa M, Suzuki N. Behcet's disease. Clin Exp M ed. 2004;4:10-20.

5. Kuzu M A, O zaslan C, Koksoy C, Gurler A, Tuzuner A. $\checkmark$ ascular involvement in Behçet's disease: 8-year audit. W orld J Surg. 1994;18:948-53.

6. C riteria for diagnosis of Behcet's disease. International Study Group for Behcet's Disease. Lancet. 1990;335:1078-80.

7. Iscan ZH. Vural KM, Bayazit M. Compelling nature of arterial manifestations in Behcet disease. J Vasc Surg. 2005;41:53-8.

8. M atsumoto T, U ekusaT, FukudaY. V asculo-Behçet'sdisease: a pathologic study of eight cases. H um Pathol. 1991;22:45-51.

9. H amza M. Large artery involvement in Behcet's disease. J R heumatol. 1987;14:554-9.

10. Koc Y, Gullu I, Akpek G, et al. Vascular involvement in Behcet's disease. J R heumatol. 1992;19:402-10.

11. al-D alaan AN, al Balaa SR, el R amahi K, et al. Behcet's disease in Saudi Arabia. J Rheumatol. 1994;21:658-61.

12. Tohme A, Aoun N, El-Rassi B, Ghayad E. Vascular manifestations of Behcet's disease. Eighteen cases among 140 patients. Joint Bone Spine. 2003;70:384-9.

13. O zeren $M$, M avioglu I, D ogan OV, Yucel E. Reoperations results of arterial involvement in Behcet's disease. Eur J V asc Endovasc Surg. 2000;20:512-9.
14. Kural-Seyahi E, Fresko I, Seyahi N, et al. The long-term mortality and morbidity of Behcet's syndrome: a 2-decade outcomesurvey of 387 patientsfollowed at a dedicated center. M edicine (Baltimore). 2003;82:60-76.

15. Calamia KT, Cohen MD, O'D uffy JD. Large vassel involovment in Behcet's disease. Proceedings of the 8th International Congress on Behcet's D isease. 19980 ct 7-9; Reggio-Emilia, Italy. Reggio Emilia: Prex SRL, 1998.

16. Kaklamani VG, Tzonou A, M arkomichelakis N, Papazoglou $S$, Kaklamanis PG. The effect of smoking on the clinical features of Adamantiades-Behcet's disease. Adv Exp Med Biol. 2003;528:323-7.

17. Ceyran H, Akcali $Y$, Kahraman C. Surgical treatment of vasculo-Behcet's sisease. A review of patientswith concomitant multipleaneurysmsand venouslesions. V asa. 2003;32:149-53.

18. Kobayashi $M$, I to $M, N$ akagawa $A$, et al. $N$ eutrophil and endothelial cell activation in the vasa vasorum in vasculoBehcet disease. H istopathology. 2000;36:362-71.

19. O zeren M, D ogan OV, D ogan S, Yucel E. True and pseudo aneurysms of coronary arteries in a patient with Behcet's disease. Eur J Cardiothorac Surg. 2004;25:465-7.

20. LeThi H uong D, W echsler $B, P$ apo $T$, et al. Arterial lesions in Behcet's disease. A study in 25 patients. J Rheumatol. 1995;22:2103-13.

21. Yazici $H$, Basaran $G, H$ amuryudan $V$, et al. The ten-year mortality in Behcet's syndrome. Br J Rheumatol. 1996;35:139-41.

22. H osakaA, M iyataT, Shigematsu H, et al. Long-term outcome after surgical treatment of arterial lesions in Behcet disease. J V asc Surg. 2005;42:116-21.

23. Saba D, Saricaoglu H, Bayram AS, et al. Arterial lesions in Behcet's disease. V asa. 2003;32:75-81.

24. T uzun $H$, Besirli K, Sayin A, et al. M anagement of aneurysms in Behcet's syndrome: an analysis of 24 patients. Surgery. 1997;121:150-6.

Correspondência:

$\mathrm{N}$ ilo M itsuru Izukawa

Av. Indianópolis, 2784, Indianópolis

CEP 04062-003 - São Paulo, SP

Tel.: (11) 5594.0134

E-mail: nilosmk.ops@terra.com.br 